\title{
Visualizing sequential compound fusion and kiss-and-run in live excitable cells
}

\author{
Lihao Ge*, Wonchul Shin*, Ling-Gang $\mathrm{Wu}^{\diamond}$
}

National Institute of Neurological Disorders and Stroke, 35 Convent Dr., Bldg. 35, Rm. 2B-1012,

Bethesda, Maryland 20892, USA

*: These authors contributed equally to this work

$\diamond$ : Corresponding author, email: wul@ninds.nih.gov

Brief title: Sequential compound fusion and kiss-and-run 
Vesicle fusion is assumed to occur at flat membrane of excitable cells. In live neuroendocrine cells, we visualized vesicle fusion at $\Omega$-shape membrane generated by preceding fusion, termed sequential compound fusion, which may be followed by fusion pore closure, termed compound kiss-and-run. These novel fusion modes contribute to vesicle docking, multivesicular release, asynchronous release, and endocytosis. We suggest modifying current models of exo-endocytosis to include these new fusion modes.

Vesicle fusion releases transmitters, hormones and peptides to mediate many physiological functions, such as synaptic transmission, fight or flight response, and controlling blood glucose level relevant to diabetes ${ }^{1,2}$. In the last half a century of studies in excitable cells, including neurons and endocrine cells, all models on release steps and modes, such as vesicle docking, fusion pore opening and closure (kiss-and-run), mono- or multi-vesicular release at single release sites, synchronized or asynchronized release, are constructed under a fundamental assumption that vesicles fuse at the flat plasma membrane $(\mathrm{PM})^{1,2}$. Despite generally accepted, this assumption has not been tested in live cells. Against this concept, sequential compound fusion (Fusion seq-comp $_{\text {) }}$ - vesicle fusion at a previously fused vesicular $\Omega$-shape structure - has long been proposed in nonexcitable cells containing extremely large $(\sim 1-5 \mu \mathrm{m})$ granules that release contents extremely

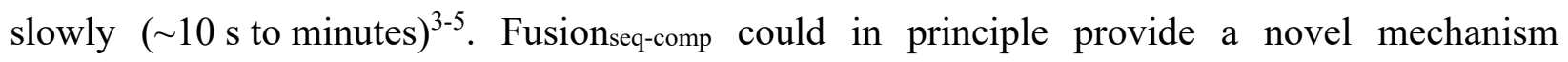
underlying a series of fusion steps and modes, such as vesicle docking, desynchronized multivesicular release, asynchronous release, and subsequent endocytosis. These fusion steps and modes may enhance synaptic strength, synaptic reliability, firing information transfer, and the dynamic range of synaptic plasticity and neuromodulation at many synapses ${ }^{1,6-8}$. However, the

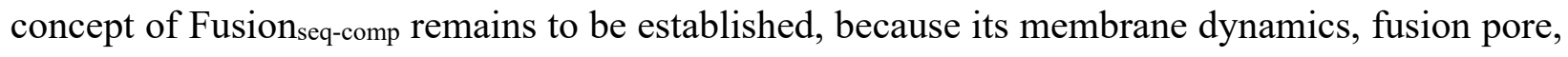


and content release have not been directly visualized and thus proved in any non-excitable or

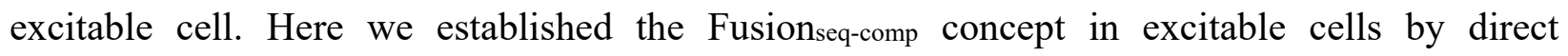
visualization of its membrane dynamics, fusion pore dynamics and content release dynamics in live cells for the first time.

To visualize membrane dynamics, we transfected EGFP or mNeonGreen attached to phospholipase $\mathrm{C}$ delta $\mathrm{PH}$ domain $\left(\mathrm{PH}_{\mathrm{G}}\right)$, which binds to $\operatorname{PtdIns}(4,5) \mathrm{P} 2\left(\mathrm{PIP}_{2}\right)$ at, and thus labels the plasma membrane (PM) in neuroendocrine cells, the primary cultured bovine adrenal chromaffin cell (Fig. 1a) $)^{9,10}$. We added Atto 532 (A532) in the bath, which enters and thus labels fusing vesicles' $\Omega$-shape profiles (Fig. 1a $)^{9,11}$. A 1-s depolarization $(-80$ to $+10 \mathrm{mV})$ via a pipette at the whole-cell configuration induced calcium currents, capacitance changes reflecting exo- and endocytosis, and fusion events observed with stimulated emission depletion (STED) microscopy of $\mathrm{PH}_{\mathrm{G}} / \mathrm{A} 532$ (Fig. 1b-e). Images were acquired at the XZ-plane (near cell bottom) with Y-location fixed at about the cell center for $\sim 1-2 \mathrm{~min}\left(\mathrm{XZ} / \mathrm{Y}_{\text {fix }}\right.$ imaging, 26-300 $\mathrm{ms}$ per frame, Fig. 1a); each cell was subjected to only 1 depol $_{1 \text { s }}$ to avoid whole-cell exo- and endocytosis run-down ${ }^{9,11}$.

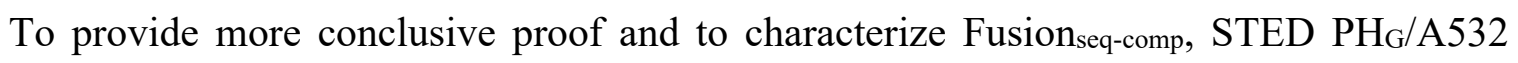
imaging data were collected from a large number of cells, 1211 cells, at the voltage-clamp configuration. A total of $336 \mathrm{PH}_{\mathrm{G}}$-labelled $\Omega$-shape profiles filled with A532 $\left(\Omega_{\mathrm{PH}}\right)$ appeared within a single image frame (26-300 ms), reflecting vesicle fusion that allowed for $\mathrm{PH}_{\mathrm{G}} / \mathrm{A} 532$ diffusion from PM/bath into the fusion-generated $\Omega$-profile (Fig. 1c-f). Among $336 \Omega_{\mathrm{PH}}, 247 \Omega_{\mathrm{PH}}$ (73.5\%) appeared at the flat PM (Fig. 1c, f), reflecting single vesicle fusion (Fusion $_{\text {single, for detail, see Ref. }}{ }^{9,10,12}$ ); $23 \Omega_{\mathrm{PH}}(6.9 \%$ ) appeared at flat PM, but followed at 0.2$85 \mathrm{~s}$ later on its top by a sudden appearance of another $\Omega \mathrm{PH}$, forming an 8-shape structure reflecting Fusion $_{\text {seq-comp }}$ (Fig. 1d, f); $66 \Omega_{\mathrm{PH}}(19.6 \%)$ appeared on the top of $\Omega_{\mathrm{PH}}$ preformed before depol $\mathrm{l}_{\mathrm{s}}$ 
$\left(\right.$ Fusion $_{\text {on_pre- } \Omega}$ ), which also formed an 8-shape structure (Fig. 1e, f). Preformed $\Omega_{\mathrm{PH}}$ could be from previous fusion events that maintained a $\Omega$-shape, as recently reported ${ }^{9,}{ }^{11}$. Supporting this possibility, the $2^{\text {nd }}$ fusion may occur $\sim 20-85 \mathrm{~s}$ after $1^{\text {st }}$ fusion during Fusion seq-comp



To demonstrate the release dynamics of Fusion $\_$seq-comp and Fusionon_pre- $\Omega$, we loaded vesicles with fluorescent false neurotransmitter FFN511, a substrate for vesicle monoamine

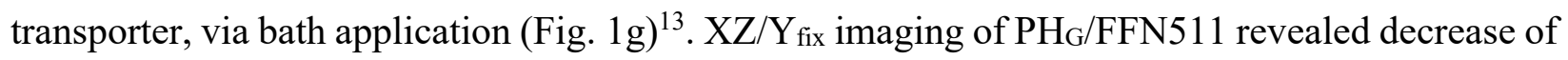
FFN511 spot fluorescence (FFF) and simultaneous appearance of $\Omega_{\mathrm{PH}}$ at the same spot, reflecting fusion-generated $\Omega$ PH that releases FFN511 (Fig. 1h-j). FFN511 releasing spots may 1) fuse on flat

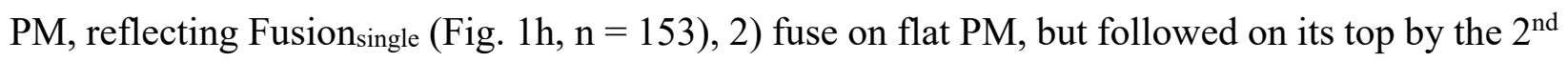
fusion that released its FFN511 content and created the $2^{\text {nd }} \Omega_{\mathrm{PH}}$, (forming an 8-

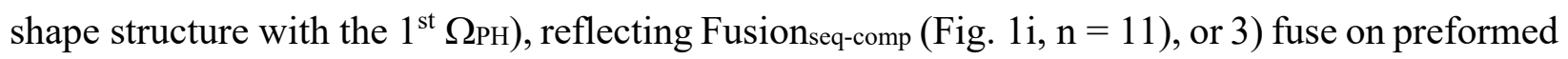
$\Omega_{\mathrm{PH}}$ to form a $\mathrm{PH}_{\mathrm{G}}$-labelled 8-shape structure, reflecting Fusion ${ }_{\mathrm{on} \_p r e-\Omega}(\mathrm{n}=31$, Fig. 1j). These results established the concept of Fusion seq-comp $_{\text {and Fusion }}$ _pre- $\Omega$ by demonstrating their vesicular positions and content release.

Next, we examined fusion pore and $\Omega_{\mathrm{PH}}$ membrane dynamics of Fusion seq-comp $_{\text {and }}$

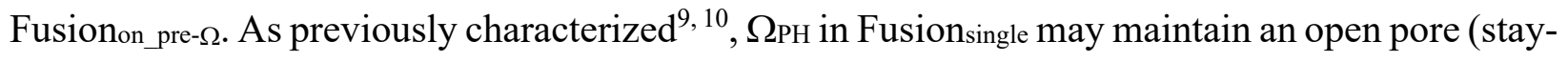
fusion, Fig. 2a), close its pore at $\sim 0.05$ - 30 s later (close-fusion, Fig. 2b), or shrink to merge with the plasma membrane (shrink-fusion, Fig. 2c; summarized in Fig. 2d). Close-fusion was detected as A532 fluorescence (F532, strongly excited) dimming due to pore closure that prevented bath fluorescent A532 from exchanging with bleached A532, while $\mathrm{PH}_{\mathrm{G}}$ fluorescence (FrH, weakly excited) sustained or decayed with a delay that reflected PtdIns(4,5) $\mathrm{P}_{2}$ conversion 
into PtdIns(4)P and/or vesicle pinch off (Fig. 2b); stay-fusion was detected as sustained $\mathrm{F}_{532}$ and $F_{P H}$ (Fig. 2a); shrink-fusion, $\Omega_{P H}$ shrinking with parallel decreases of $F_{532}$ and $F_{P H}$ (Fig. 2c) (for detail, see Refs. ${ }^{9,10}$ ).

The $2^{\text {nd }} \Omega_{\mathrm{PH}}$ in Fusion seq-comp $_{\text {and Fusionon_pre- } \Omega}$ may 1) remain unchanged with an open pore, reflected as sustained F532 and FPH (Fig. 2e, f), analogous to Fusionsingle's stay-fusion (Fig. 2a), 2) close its pore at $\sim 0.05-30 \mathrm{~s}$ later, reflected as F532 decay while FrH sustained or

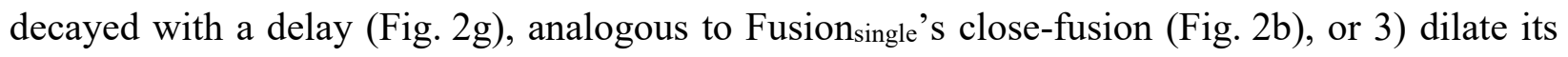
pore till the 8-shape was converted to an elongated or large $\Omega$-shape (e.g., Fig. 2h, i; Fig. 2 j shows their percentages). We termed $2^{\text {nd }} \Omega_{\mathrm{PH}}$ close-fusion (e.g., Fig. 2 g)

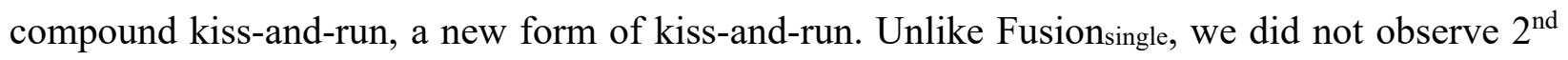
$\Omega_{\mathrm{PH}}$ shrink-fusion, but pore dilation (Fig. 2h-j).

The $20-80 \%$ FFN511 fluorescence decay time was similar between the $2^{\text {nd }}$ and the $1^{\text {st }} \Omega_{\mathrm{PH}}$

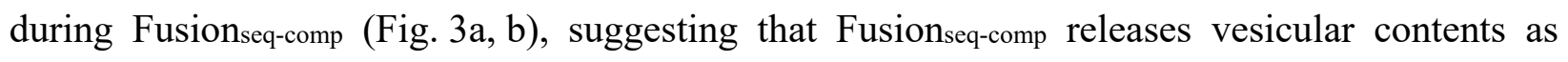

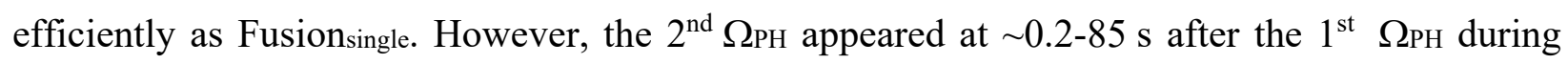

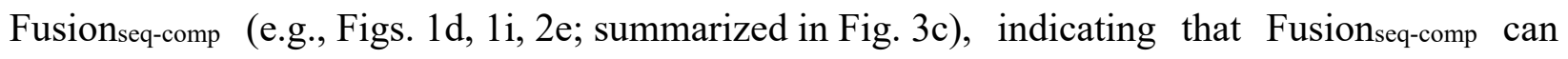
generate desynchronized multi-vesicle release at single release sites. Given that the $1^{\text {st }} \Omega \mathrm{PH}$ occurred mostly during and within $\sim 1 \mathrm{~s}$ after depol1s $\left(\right.$ Fig. 3c), the $1^{\text {st }} \Omega$ PH reflected synchronized release; the various time delay of the $2^{\text {nd }} \Omega_{\mathrm{PH}}$ (Fig. 3c) thus reflected asynchronous release. We

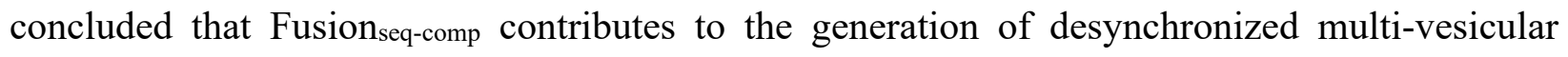
release and asynchronous release.

The present work firmly established the concept of sequential compound fusion and compound kiss-and-run by directly visualizing their membrane, fusion pore and content-release 
dynamics in live cells for the first time. Establishing these new concepts in excitable cells may conceptually advance our understanding of secretory vesicle exo-endocytosis, because sequential compound fusion and compound kiss-and-run may constitute new mechanisms contributing to the generation of desynchronized multi-vesicular release at single release sites, asynchronous release, vesicle docking and priming, and vesicle endocytosis, as discussed below.

Multi-vesicular release from single release sites enhance synaptic strength, synaptic reliability, and the dynamic range of synaptic plasticity and neuromodulation at many synapses ${ }^{1}$. The mechanism underlying multi-vesicular release at single release sites is poorly understood ${ }^{1}$. Sequential compound fusion readily explains how single release sites produce multi-vesicular release, particularly the desynchronized multi-vesicular release, which enhances the precise and efficient firing information transfer at synapses ${ }^{6,7}$. If the release interval of sequential compound fusion is minimal, it may also explain the coordinated or simultaneous multi-vesicular release at single release sites $^{1,14}$.

While depolarization-evoked release is mostly synchronized, asynchronous release lasting much longer than the brief depolarization also takes place in many excitable cells, which may transfer a brief presynaptic firing burst into a prolonged postsynaptic firing burst at synapses ${ }^{8}$. The differences in the mechanisms underlying asynchronous and synchronous release remain not well understood. Different calcium sensors with different calcium affinity have been suggested ${ }^{8}$. Sequential compound fusion evidently generates a delay in releasing the second vesicle (Fig. 3), providing a novel mechanism contributing to the generation of asynchronous release.

Vesicle fusion must involve vesicle movement towards the PM release sites for docking and subsequent vesicular V-SNARE and PM T-SNARE binding that may prime docked vesicles for release ${ }^{8,15}$. Our findings suggest modifying this concept by including a new mechanism of 
generating release sites for docking - the fusion-generated $\Omega$-profiles are maintained and serve as the new release sites for docking and priming. Such a docking process saves vesicles from spending energy to travel one-vesicle-length of distance for docking at the flat PM. The priming process may involve diffusion of T-SNARE from the PM to the fusion-generated vesicular $\Omega$ profile and T-SNARE binding with V-SNARE of the docking vesicle ${ }^{16}$. These processes may take more time ${ }^{16}$, explaining the prolonged release interval of sequential compound fusion that produces asynchronous release.

Compound kiss-and-run reported here is a new mode of exo-endocytosis that retrieves vesicles undergoing sequential compound fusion. It may explain the electron microscopic observation of 8-shape (or sausage-shape) structures and large vesicles that are otherwise interpreted as different mechanisms, such as vesicle budding, bulk endocytosis of large vesicles, and/or cytosol vesicle-vesicle fusion. We suggest modifying current models of secretory vesicle endocytosis $^{2,17}$ by including compound kiss-and-run as a new mode of endocytosis.

While obtained from chromaffin cells containing large dense-core vesicles, our findings are most likely applicable to neurons for two reasons. First, like neurons, chromaffin cells are excitable cells with a neuronal origin and very similar calcium-, synaptotagmin-, SNARE-, and dynamin-dependent exo- and endocytosis ${ }^{2,18}$. Second, neuron contains large dense-core and small clear-core vesicles, both of which may maintain an $\Omega$-shape after fusion ${ }^{19-22}$, the prerequisite for mediating sequential compound fusion. Direct visualization is needed to prove sequential compound fusion and kiss-and-run in neurons. Visualizing sequential compound fusion of small synaptic vesicles $(\sim 30-50 \mathrm{~nm})$ requires much higher spatial-temporal resolution than what we have ( $\sim 60 \mathrm{~nm} / 26-300 \mathrm{~ms})$. Significant technical advancements are needed to overcome this technical problem in the future. 
Cytosolic vesicle-vesicle fusion is the first form of compound fusion being proposed, based on the observation of 1) cytosolic 8-shape or sausage-like vesicular structures, and 2) capacitance jumps and synaptic miniature currents (quantal size) too large for single vesicle fusion in nonexcitable and excitable cells, such as pancreatic acinar cells, eosinophils, mast cells, calyx-type and ribbon-type synapses ${ }^{3,5,23-27}$. However, direct observation of the membrane transformation during vesicle-vesicle fusion, which can fully establish the concept of vesicle-vesicle fusion is still missing. Similar to vesicle-vesicle fusion, sequential compound fusion was suggested in nonexcitable cells based on the observation of 1) sequential release of lysotracker green loaded into the extremely large $(\sim 1-5 \mu \mathrm{m})$ granules in eosinophils ${ }^{3}$, and 2) sequential generation of extracellular-dye-loaded extremely large tube-like structures from the PM into the cytosol in acinar cells ${ }^{4}$. However, these observations could not fully exclude the possibility that the extremely large, apparently cytosolic structure could be docked at PM that was not visible at the imaged plane, or that the extracellular-dye-loaded structure reflects endocytic membrane invagination (content release is not imaged simultaneously). Because of these uncertainties, and most importantly the lack of evidence showing direct, simultaneous membrane, pore and release dynamics of sequential compound fusion in live cells, the concept of sequential compound fusion is not fully established ${ }^{2}$. The present work provided the missing evidence required to fully establish the concept of sequential compound fusion - the dynamics of membrane transformation, fusion pore, and content release. Furthermore, we link this concept to a new endocytic mode, compound kiss-and-run, and extend these concepts to excitable cells that release much smaller vesicles rapidly. Thus, sequential compound fusion and compound kiss-and-run may be a widespread exoendocytosis mode used by excitable and non-excitable cells to release vesicular contents that may mediate important functions such as neuronal communication, fight or flight response, regulation 
of blood glucose level relevant to diabetes, and immune responses ${ }^{1,2}$. The technique we used here opens the door to study the functions and mechanisms of sequential compound fusion in live cells.

Acknowledgements: This work was supported by the National Institute of Neurological Disorders and Stroke Intramural Research Program (ZIA NS003009-15 and ZIA NS003105-10) to L.G.W. We thank Carolyn Smith for STED microscopy support.

Author Contributions: L.G. did most FFN511-related experiments. W.S. did most PHG/A532related experiments. L.G.W. supervised the project. L.G. wrote the experimental results; L.G.W. wrote the manuscript with help from L.G. and W.S.

Declaration of interests: The authors declare no competing interests. 


\section{Figure Legends}

Figure 1. Visualizing sequential compound fusion and fusion on pre-formed $\Omega$-profiles in

\section{live cells}

a, Left: setup drawing. Cell's membrane is labelled with $\mathrm{PH}_{\mathrm{G}}$ (green), and bath labelled with A532 (red). ICa and Cm (capacitance) are recorded from the whole-cell pipette.

Right: XZ-plane $\mathrm{PH}_{\mathrm{G}}$ (green) and A532 images for a fraction of a cell (near cell bottom) at rest. Cytosol, PM and coverslip locations are labelled.

b, Sampled ICa and Cm change induced by depol $1_{1}$.

c-e, $\Omega_{\mathrm{PH}} \quad$ fluorescence $\quad\left(\mathrm{FPH}_{\mathrm{PH}}\right.$, normalized to baseline), A532 spot fluorescence (F532, normalized to baseline) and STED $\mathrm{XZ} / \mathrm{Y}_{\text {fix }}$ images at times indicated with lines for

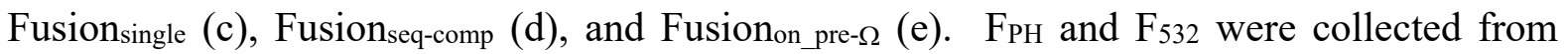
fusing vesicle(s).

f, The percentage of Fusion single, Fusion $_{\text {seq-comp }}$ and Fusion on_pre- $\Omega_{\Omega}$ observed with STED XZ/Y fix imaging of $\mathrm{PHG} / \mathrm{A} 532$ (336 events from 274 cells showing these fusion events).

g, Similar to panel a, but for imaging FFN511-loaded vesicles (purple, pseudo-colour) and PHGlabelled cell membrane (green).

h-j, FPH, FFN511 spot fluorescence $\left(F_{F F N}\right)$ and STED XZ/Y fix images at times indicated with lines showing release of FFN511 for Fusion single $_{\text {(g), }} \quad$ Fusion $_{\text {seq-comp }}$ (h, vesicle 1 and 2 are circled and labelled), and Fusion $\_$_pre- $\Omega(\mathrm{j})$. 


\section{Figure 2. Pore dynamics of sequential compound fusion and fusion on pre-formed $\Omega$-profiles}

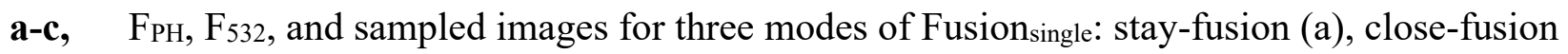
(b), and shrink-fusion (c).



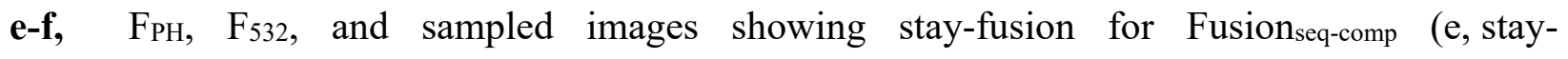
fusion refers to $2^{\text {nd }}$ fusion event) and for Fusionon_pre- $\Omega$ (f).

g, FPH, F532, and sampled images showing close-fusion for Fusionon_pre- $\Omega$.

h, FPH, F532, and sampled images showing dilation of the fusion pore during Fusion seq-comp $_{\text {s. }}$ (pore dilation refers to $2^{\text {nd }}$ vesicle fusion)

i, $\mathrm{F}_{\mathrm{PH}}, \mathrm{F}_{511}$, and sampled images showing dilation of the fusion pore during Fusion

j, Percentage of stay-fusion, close-fusion and pore dilation for the vesicle that fused at the previously generated $\Omega_{\mathrm{PH}}(\mathrm{n}=89)$. Data from Fusion seq-comp $_{(\mathrm{n}=23) \text { and Fusionon_pre- } \Omega}(\mathrm{n}=66)$ were pooled together.

\section{Figure 3. Sequential compound fusion releases contents efficiently, but generates} desynchronized multi-vesicular release and asynchronized release at single release sites

a, FPH, FFFN, and sampled images of the $1^{\text {st }}$ (dotted circle, dotted trace) and the $2^{\text {nd }}$

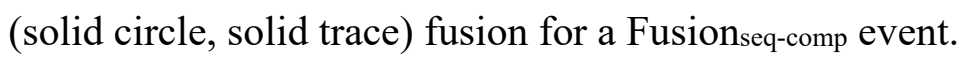

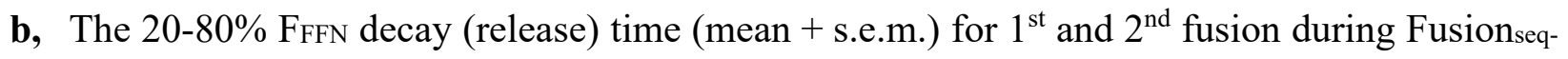
comp (11 events, 11 cells under $\mathrm{PH}_{\mathrm{G}} / \mathrm{FFN} 511$ imaging). No significant difference was found (paired t test, $\mathrm{p}=0.712$ ). 
c, Upper: the onset time of the $1^{\text {st }}$ and the $2^{\text {nd }}$ fusion during Fusion seq-comp (23 events, 23 cells under $\mathrm{PH}_{\mathrm{G}} / \mathrm{A} 532$ imaging). 1 fusion per circle; dash lines connect two

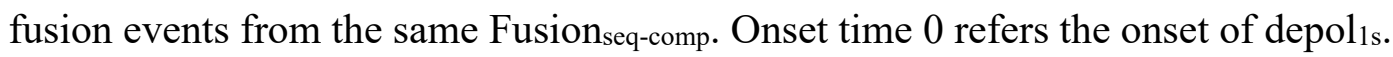

Lower: mean onset $(+$ s.e.m., $n=23)$ of the $1^{\text {st }}$ and the $2^{\text {nd }}$ fusion during Fusion seq-comp $_{\text {s. }}$ $(* *: \mathrm{p}<0.01$, paired t-test). Left and right panels are from the same data set. 


\section{Materials and Methods}

\section{Chromaffin cell culture}

We prepared primary bovine adrenal chromaffin cell culture as described previously ${ }^{11}$. Fresh adult (21 - 27 months old) bovine adrenal glands (from a local abattoir) were immersed in pre-chilled Locke's buffer on ice containing: NaCl, 145 mM; KCl, 5.4 mM; Na2HPO4, 2.2 mM; NaH2PO4, $0.9 \mathrm{mM}$; glucose, $5.6 \mathrm{mM}$; HEPES, $10 \mathrm{mM}$ (pH 7.3, adjusted with $\mathrm{NaOH}$ ). Glands were perfused with Locke's buffer, then infused with Locke's buffer containing collagenase P $(1.5 \mathrm{mg} / \mathrm{ml}$, Roche $)$, trypsin inhibitor $(0.325 \mathrm{mg} / \mathrm{ml}$, Sigma $)$ and bovine serum albumin ( $5 \mathrm{mg} / \mathrm{ml}$, Sigma), and incubated at $37^{\circ} \mathrm{C}$ for $20 \mathrm{~min}$. The digested medulla was minced in Locke's buffer, and filtered through a $100 \mu \mathrm{m}$ nylon mesh. The filtrate was centrifuged (48 xg, $5 \mathrm{~min}$ ), re-suspended in Locke's buffer and re-centrifuged until the supernatant was clear. The final cell pellet was re-suspended in pre-warmed DMEM medium (Gibco) supplemented with 10\% fetal bovine serum (Gibco).

\section{Electroporation and plating}

Cells were transfected by electroporation using Basic Primary Neurons Nucleofector Kit (Lonza), according to the manufacturer's protocol and plated onto glass coverslips with mouse Laminin coating over PDL layer (Neuvitro). The cells were incubated at $37^{\circ} \mathrm{C}$ with $9 \% \mathrm{CO} 2$ and used within 5 days.

\section{Plasmids and fluorescent dyes}

The PH-EGFP (phospholipase C delta PH domain attached with EGFP) was obtained from Dr. Tamas Balla. PH-mNeonGreen construct was created by replacing the EGFP tag of PH-EGFP with mNeonGreen (Allele Biotechnology) ${ }^{28}$. Both PH-EGFP and PH-mNeonGreen are abbreviated as 
$\mathrm{PH}_{\mathrm{G} .}$ For Atto 532 (A532, Sigma) imaging, A532 concentration in the bath solution was $30 \mu \mathrm{M}$. For FFN511 (Abcam) imaging, cells were bathed with FFN511 (5-10 $\mu \mathrm{M})$ for 10 min and images were performed after washing out FFN511 in the bath solution.

Overexpression of $\mathrm{PH}_{\mathrm{G}}$ did not significantly affect the basic properties of exo- and endocytosis, because 1) whole-cell capacitance measurements and imaging show robust exo- and endocytosis, and similar percentages of close-fusion and non-close-fusion as control ${ }^{10,11}$, and 2) $\mathrm{PH}_{\mathrm{G}}$-labelled fusion pore could also be observed with imaging of extracellularly applied mCLINGA488 or with $\mathrm{EM}^{9}$.

\section{Electrophysiology}

At room temperature $\left(20-22^{\circ} \mathrm{C}\right)$, whole-cell voltage-clamp and capacitance recordings were performed with an EPC-10 amplifier together with the software lock-in amplifier (PULSE, HEKA, Lambrecht, Germany) ${ }^{11,29}$. The holding potential was $-80 \mathrm{mV}$. For capacitance measurements, the frequency of the sinusoidal stimulus was $1000-1500 \mathrm{~Hz}$ with a peak-to-peak voltage $\leq 50 \mathrm{mV}$. The bath solution contained $125 \mathrm{mM} \mathrm{NaCl}, 10 \mathrm{mM}$ glucose, $10 \mathrm{mM}$ HEPES, $5 \mathrm{mM} \mathrm{CaCl}_{2}, 1 \mathrm{mM} \mathrm{MgCl} 2,4.5 \mathrm{mM} \mathrm{KCl}$, and $20 \mathrm{mM}$ TEA, $\mathrm{pH} 7.3$ adjusted with $\mathrm{NaOH}$. The pipette $(2-4 \mathrm{M} \Omega)$ solution contained $130 \mathrm{mM}$ Cs-glutamate, $0.5 \mathrm{mM}$ Cs-EGTA, $12 \mathrm{mM} \mathrm{NaCl}$, $30 \mathrm{mM}$ HEPES, $1 \mathrm{mM} \mathrm{MgCl} 2,2 \mathrm{mM}$ ATP, and $0.5 \mathrm{mM}$ GTP, pH 7.2 adjusted with CsOH. These solutions pharmacologically isolated calcium currents.

For stimulation, we used a 1-s depolarization from the holding potential of $-80 \mathrm{mV}$ to +10 $\mathrm{mV}$ (depol1s). We used this stimulus, because it induces robust exo-endocytosis as reflected in capacitance recordings (Fig. 1a) ${ }^{11,30,31}$. In a fraction of experiments during FFN511 imaging, we 
used 10 pulses of $400-\mathrm{ms}$ depolarization from $-80 \mathrm{mV}$ to $+10 \mathrm{mV}$ at $2 \mathrm{~Hz}$, which evoked more fusion events.

\section{STED imaging}

STED images were acquired with Leica TCS SP8 STED $3 \times$ microscope that is equipped with a $100 \times 1.4$ NA HC PL APO CS2 oil immersion objective and operated with the LAS-X imaging software. Excitation was with a tunable white light laser and emission was detected with hybrid detectors. In time-gated STED mode, PH-EGFP and A532 were sequentially excited at 470 and $532 \mathrm{~nm}$, respectively, with the $592 \mathrm{~nm}$ STED depletion beam, and their fluorescence collected at 475-525 nm and 540-587 nm, respectively. PH-mNeonGreen and A532 were sequentially excited at 485 and $540 \mathrm{~nm}$, respectively, with the $592 \mathrm{~nm}$ STED depletion beam, and their fluorescence collected at 490-530 $\mathrm{nm}$ and 545-587 nm, respectively. PH-mNeonGreen and FFN511 were sequentially excited at 505 and $442 \mathrm{~nm}$, respectively, with the $592 \mathrm{~nm}$ STED depletion beam, and their fluorescence collected at 510-587 $\mathrm{nm}$ and $447-490 \mathrm{~nm}$, respectively.

The excitation power for A532 was 10\% of the maximum, at which fluorescent A532 can be bleached within a few seconds. This feature was used to distinguish whether the fusion pore is closed or not, because pore closure prevents bleached A532 (caused by strong excitation) from exchange with fluorescent A532 in the bath, resulting in A532 spot fluorescence decay ${ }^{9-11}$. In contrast, an open pore would not cause A532 spot fluorescence decay, because an open pore allows for continuous exchange of bleached A532 in the $\Omega$-profile with fluorescent A532 in the bath ${ }^{9-11}$.

STED imaging generally causes more photobleaching and phototoxicity. Severe phototoxicity could cause loss of the whole-cell giga seal during patch-clamp recording ${ }^{11}$. In general, we avoided severe phototoxicity by applying only one depol $1_{\mathrm{s}}$ and imaging for $\sim 1-2 \mathrm{~min}$ 
per cell. With this setting, we have not noticed significant differences in the exo- and endocytosis properties obtained under confocal and STED imaging conditions ${ }^{10,11}$. For imaging of $\mathrm{PH}_{\mathrm{G}}$ and A532, continuous exchange of bleached $\mathrm{PH}_{\mathrm{G}}$ or $\mathrm{A} 532$ with fluorescent ones from non-imaging areas lessened the photobleaching problem.

\section{STED scanning modes}

STED images were acquired at the cell bottom at the XZ-plane (perpendicular to the coverslip) with the Y-axis location fixed at about the cell center (Figure 1a, XZ/Y fix scanning mode). We repeated $\mathrm{XZ} / \mathrm{Y}_{\text {fix }}$ scanning every $26-300 \mathrm{~ms}$ at $15 \mathrm{~nm}$ per pixel in an $\mathrm{XZ}$ area of $19.4 \mu \mathrm{m} \times$ 0.7$1.5 \mu \mathrm{m}^{9}$

The STED resolution for imaging $\mathrm{PH}_{\mathrm{G}}$ (PH-EGFP or PH-mNeonGreen) in our conditions was $\sim 60 \mathrm{~nm}$ on the microscopic $\mathrm{X}$ - and $\mathrm{Y}$-axis (parallel to cell-bottom membrane or coverslip), and $\sim 150-200 \mathrm{~nm}$ on the microscopic Z-axis. STED images were deconvolved using Huygens software (Scientific Volume Imaging).

\section{Data selection}

For every cell recorded with a pipette under the whole-cell configuration, the data within the first $2 \mathrm{~min}$ at the whole-cell configuration were used, which avoided rundown of endocytosis (gradual disappearance of endocytosis) as previously reported under the whole-cell configuration for a long time ${ }^{11,32}$. Cells expressed with $\mathrm{PH}_{\mathrm{G}}$ were used for visualization of fusion events. The criteria for selecting $\mathrm{PH}_{\mathrm{G}}$-labelled $\Omega$ for analysis during XZ scanning are described in Figure $\mathrm{S} 2$ of Shin et al., 2018. 


\section{Analysis of $\mathrm{PH}_{\mathrm{G}}$-labelled $\Omega$-shape profiles}

STED images of PH- $\Omega$ were analyzed with ImageJ and LAS X (Leica). During XZ scanning, some depols-induced PH- $\Omega$-profiles were out of the same Y focal plane, as the outline of the $\Omega$-profile was vague or unclear (for detail, see ${ }^{9}$ ). These out-of-focus $\Omega$-profiles were not included for analysis.

Pores labelled with $\mathrm{PH}_{\mathrm{G}}$ were identified based on the image and the fluorescence intensity line profile (for detail, see ${ }^{9}$ ). We first identified the fluorescently labelled $\Omega$-profiles with an open pore, the edge of which was continuous with PM. The intensity line profile in the pore region should show a valley with a peak at least three times larger than the baseline fluctuation (standard deviation) in the non-pore region (for detail, see ${ }^{9}$ ). The full-width-half-maximum of the valley of the intensity line profile across the pore was proportional to the pore diameter, as shown with simulation ${ }^{9}$. Pore dilation of the fusing $\Omega_{\mathrm{PH}}$ during Fusion seq-comp $_{\text {or Fsuion }}$ pre- $\Omega$ was judged with eyes.

Identification of stay-, close- and shrink-fusion during $\mathrm{XZ} / \mathrm{Y}_{\text {fix }}$ STED imaging of $\mathrm{PH}_{\mathrm{G}} / \mathrm{A} 532$ were described in detail previously ${ }^{9}$. During $\mathrm{XZ} / \mathrm{Y}_{\text {fix }}$ imaging, $\mathrm{A} 532$ was excited at a high laser power so that fluorescent A532 can be bleached with a time constant of 1.5-3.5 s. Pore closure was identified as the gradual dimming of the A532 spot fluorescence to baseline during $\mathrm{XZ} \mathrm{PH}_{\mathrm{G}} / \mathrm{A} 532$ imaging while $\mathrm{PH}_{\mathrm{G}}$ image remained unchanged or dimmed gradually without changing the $\Omega$ PH size ${ }^{9}$. A532 fluorescence dimming was not due to a narrow pore smaller than A532 molecule size, because after A532 spot dimming, bath application of an acid solution cannot quench the pH-sensitive VAMP2-EGFP or VAMP2-pHluorin overexpressed at the same spot, indicating that the spot is impermeable to $\mathrm{H}^{+}$or $\mathrm{OH}^{-}$, the smallest molecules, and thus is closed ${ }^{10}$, 11. 


\section{Statistical tests}

Data were expressed as mean \pm s.e.m. Replicates are indicated in results and figure legends. $\mathrm{N}$ represents the number of cells, fusion events, pores, or $\Omega$-profiles as indicated in results and figure legends. The statistical test used is $t$ test or ANOVA. Although the statistics were performed based on the number of cells, fusion events, pores, and $\Omega$-profiles, each group of data was collected from at least four primary chromaffin cell cultures. Each culture was from at least two glands from one bovine. 


\section{References}

1. Rudolph, S., Tsai, M.C., von Gersdorff, H. \& Wadiche, J.I. The ubiquitous nature of multivesicular release. Trends Neurosci 38, 428-438 (2015).

2. Wu, L.G., Hamid, E., Shin, W. \& Chiang, H.C. Exocytosis and endocytosis: modes, functions, and coupling mechanisms. Annu. Rev. Physiol 76, 301-331 (2014).

3. Hafez, I., Stolpe, A. \& Lindau, M. Compound exocytosis and cumulative fusion in eosinophils. J Biol Chem 278, 44921-44928 (2003).

4. Nemoto, T. et al. Sequential-replenishment mechanism of exocytosis in pancreatic acini. Nat Cell Biol 3, 253-258 (2001).

5. Pickett, J.A. \& Edwardson, J.M. Compound exocytosis: mechanisms and functional significance. Traffic 7, 109-116 (2006).

6. Auger, C., Kondo, S. \& Marty, A. Multivesicular release at single functional synaptic sites in cerebellar stellate and basket cells. J. Neuorsci 18, $4532-4547$ (1998).

7. Rudolph, S., Overstreet-Wadiche, L. \& Wadiche, J.I. Desynchronization of multivesicular release enhances Purkinje cell output. Neuron 70, 991-1004 (2011).

8. Kaeser, P.S. \& Regehr, W.G. Molecular mechanisms for synchronous, asynchronous, and spontaneous neurotransmitter release. Annu. Rev. Physiol 76, 333-363 (2014).

9. Shin, W. et al. Visualization of Membrane Pore in Live Cells Reveals a Dynamic-Pore Theory Governing Fusion and Endocytosis. Cell 173, 934-945 (2018).

10. Zhao, W.D. et al. Hemi-fused structure mediates and controls fusion and fission in live cells. Nature 534, 548-552 (2016).

11. Chiang, H.C. et al. Post-fusion structural changes and their roles in exocytosis and endocytosis of dense-core vesicles. Nat. Commun 5, 3356 (2014).

12. Shin, W. et al. Vesicle Shrinking and Enlargement Play Opposing Roles in the Release of Exocytotic Contents. Cell Rep 30, 421-431 e427 (2020).

13. Gubernator, N.G. et al. Fluorescent false neurotransmitters visualize dopamine release from individual presynaptic terminals. Science 324, 1441-1444 (2009).

14. Singer, J.H., Lassova, L., Vardi, N. \& Diamond, J.S. Coordinated multivesicular release at a mammalian ribbon synapse. Nat. Neurosci 7, 826-833 (2004).

15. Jahn, R. \& Fasshauer, D. Molecular machines governing exocytosis of synaptic vesicles. Nature 490, 201-207 (2012).

16. Gandasi, N.R. \& Barg, S. Contact-induced clustering of syntaxin and munc18 docks secretory granules at the exocytosis site. Nat Commun 5, 3914 (2014).

17. Gan, Q. \& Watanabe, S. Synaptic Vesicle Endocytosis in Different Model Systems. Front Cell Neurosci 12, 171 (2018).

18. Sharma, S. \& Lindau, M. The fusion pore, 60 years after the first cartoon. FEBS Lett 592, 3542-3562 (2018).

19. Ceccarelli, B., Hurlbut, W.P. \& Mauro, A. Turnover of transmitter and synaptic vesicles at the frog neuromuscular junction. J. Cell Biol 57, 499-524 (1973).

20. Wen, P.J. et al. Actin dynamics provides membrane tension to merge fusing vesicles into the plasma membrane. Nat. Commun 7, 12604 (2016).

21. Watanabe, S. et al. Ultrafast endocytosis at mouse hippocampal synapses. Nature 504, 242247 (2013). 
22. Persoon, C.M. et al. Pool size estimations for dense-core vesicles in mammalian CNS neurons. EMBO J 37 (2018).

23. Ichikawa, A. Fine Structural Changes in Response to Hormonal Stimulation of the Perfused Canine Pancreas. J Cell Biol 24, 369-385 (1965).

24. Alvarez, d.T. \& Fernandez, J.M. Compound versus multigranular exocytosis in peritoneal mast cells. J Gen. Physiol 95, 397-409 (1990).

25. Scepek, S. \& Lindau, M. Focal exocytosis by eosinophils--compound exocytosis and cumulative fusion. EMBO J 12, 1811-1817 (1993).

26. He, L. et al. Compound vesicle fusion increases quantal size and potentiates synaptic transmission. Nature 459, 93-97 (2009).

27. Matthews, G. \& Sterling, P. Evidence that vesicles undergo compound fusion on the synaptic ribbon. J. Neurosci 28, 5403-5411 (2008).

28. Shaner, N.C. et al. A bright monomeric green fluorescent protein derived from Branchiostoma lanceolatum. Nat. Methods 10, 407-409 (2013).

29. Lindau, M. \& Neher, E. Patch-Clamp Techniques for Time-Resolved Capacitance Measurements in Single Cells. Pflugers Archiv-European Journal of Physiology 411, 137146 (1988).

30. Perrais, D., Kleppe, I.C., Taraska, J.W. \& Almers, W. Recapture after exocytosis causes differential retention of protein in granules of bovine chromaffin cells. J. Physiol 560, 413428 (2004).

31. Engisch, K.L. \& Nowycky, M.C. Compensatory and excess retrieval: two types of endocytosis following single step depolarizations in bovine adrenal chromaffin cells. $J$ Physiol 506 ( Pt 3), 591-608 (1998).

32. Smith, C. \& Neher, E. Multiple forms of endocytosis in bovine adrenal chromaffin cells. J. Cell Biol 139, 885-894 (1997). 

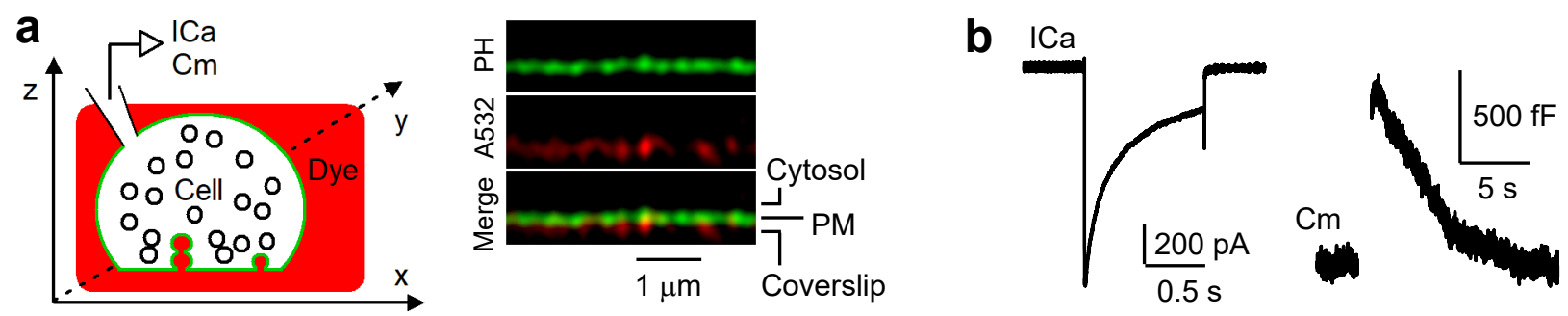

C

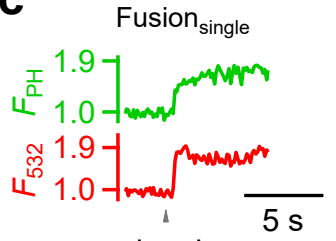

d
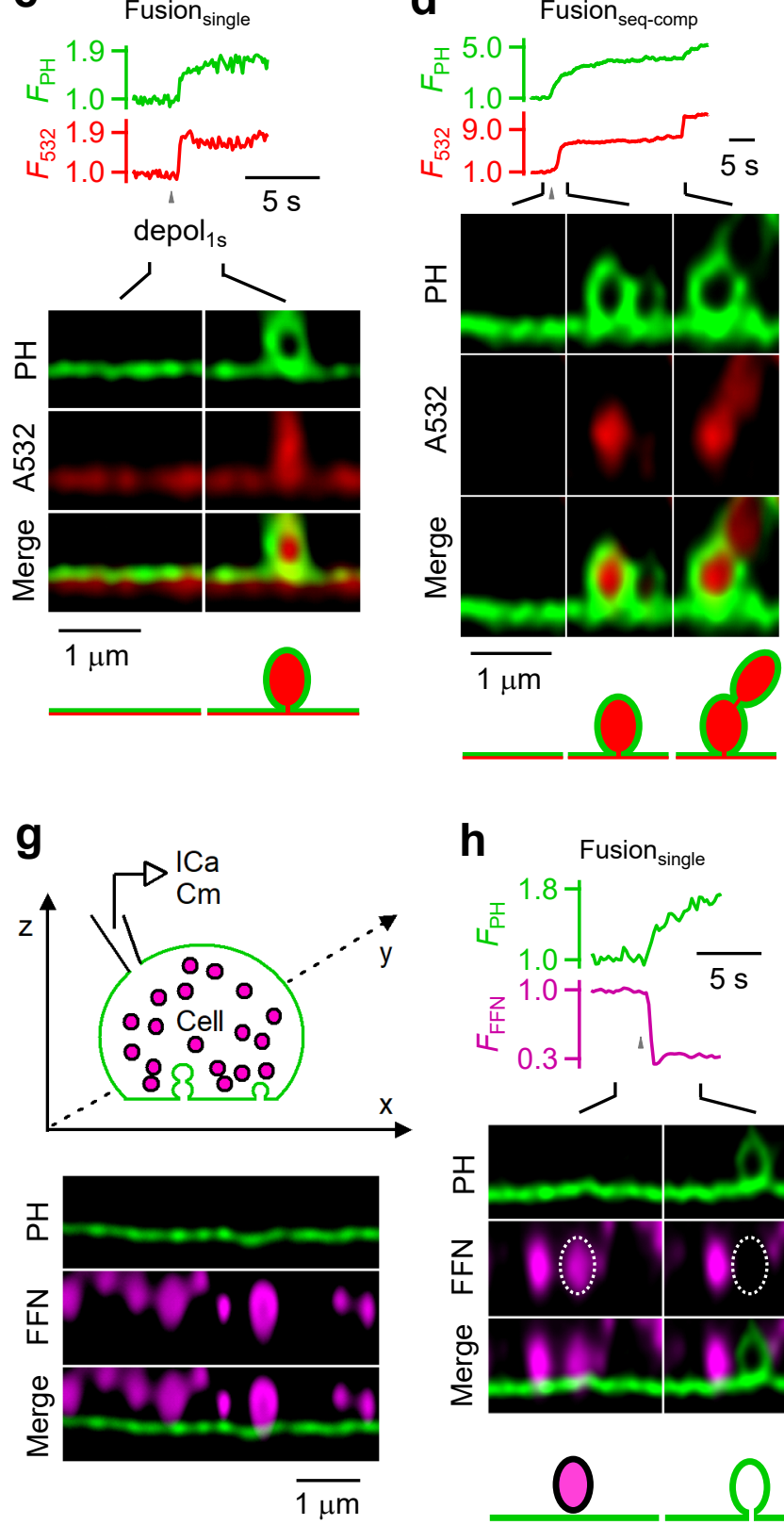

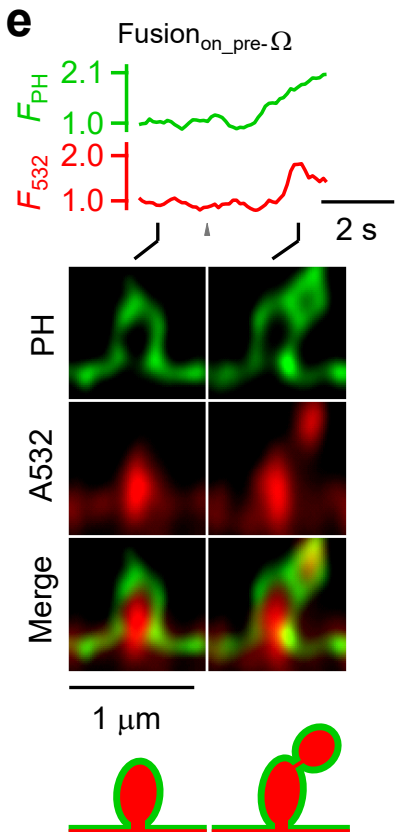

f

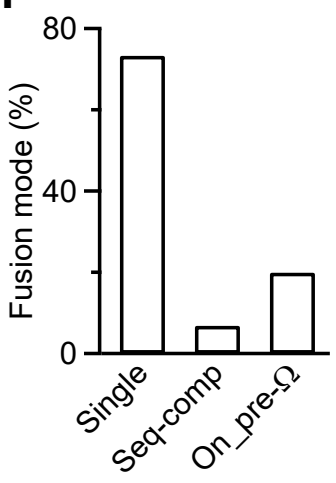

i Fusion $_{\text {seq-comp }}$

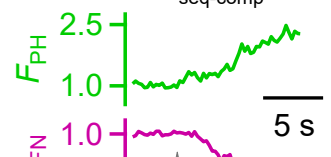

j Fusion ${ }_{\text {on_pre- } \Omega}$

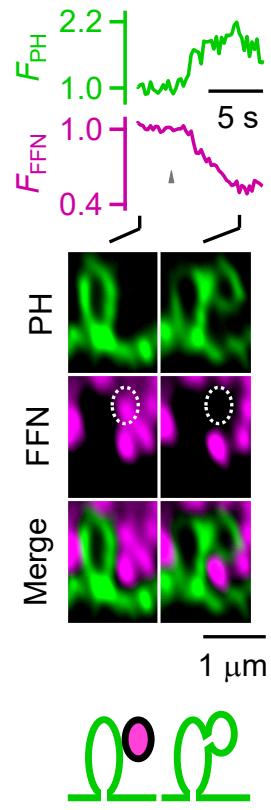

h Fusion $_{\text {single }}$

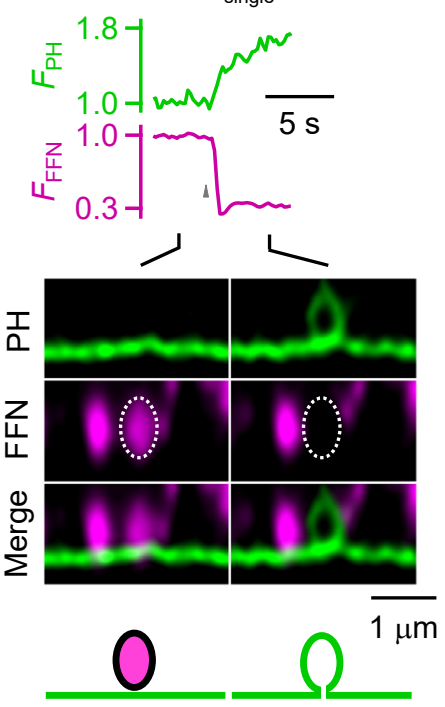

Figure 1 

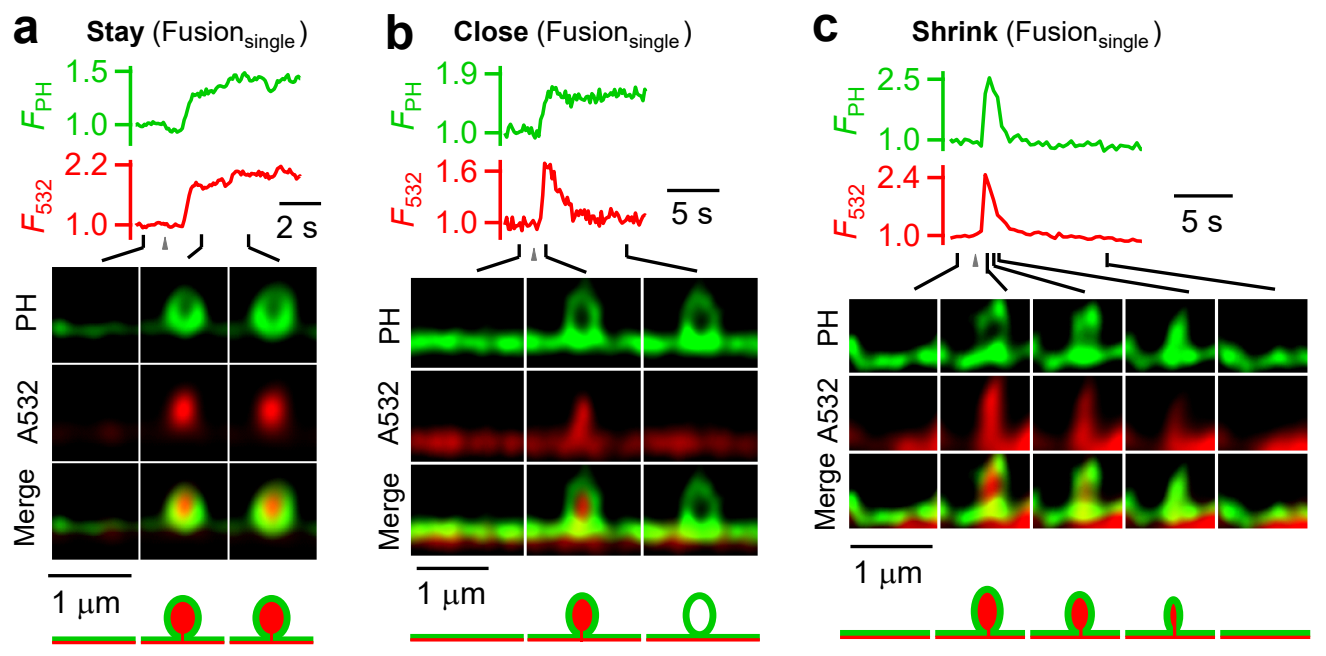

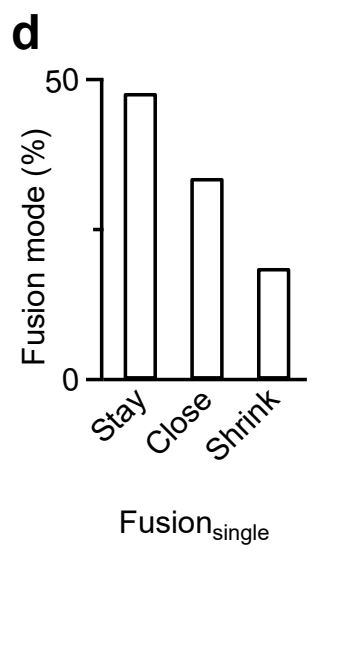

f Stay $\left(\right.$ Fusion $\left._{\text {on_pre- } \Omega} \Omega\right)$

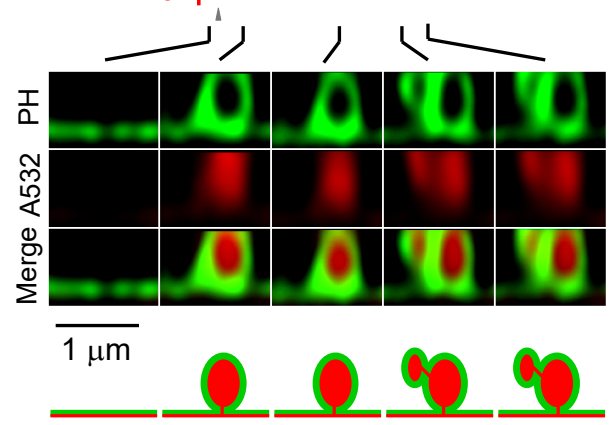

d g Close (Fusion $\_$on_pre- $\Omega$ )
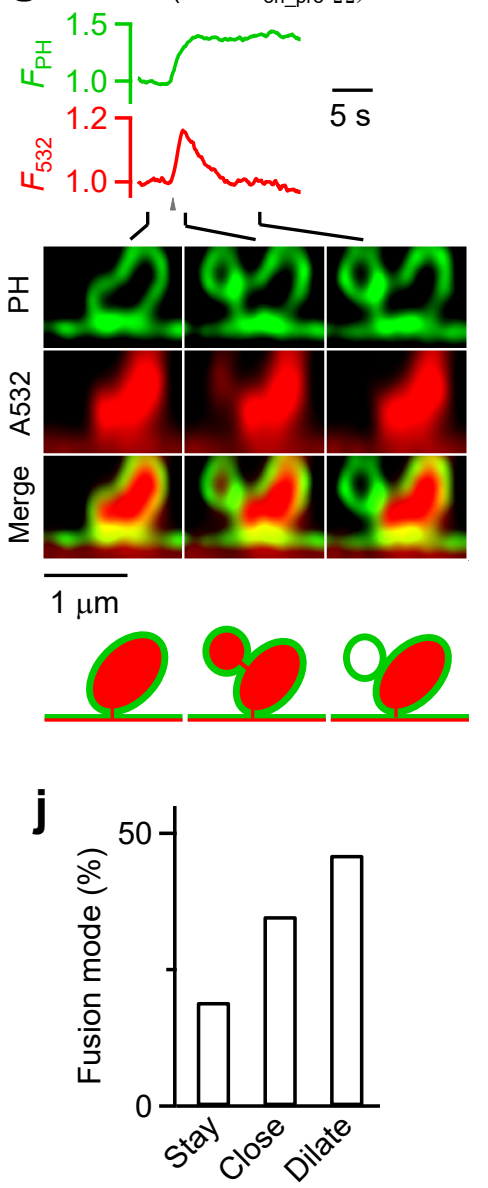

Fusion $_{\text {seq-comp }}+$ Fusion $_{\text {on_pre- } \Omega} \Omega$

$$
\text { (1) }
$$

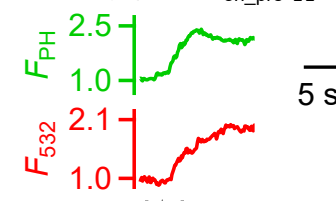

h Dilate (Fusion seq-comp $_{\text {) }}$ )
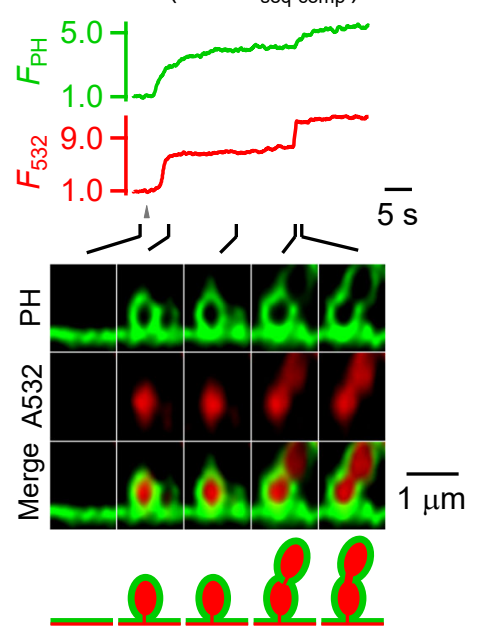

i Dilate (Fusion ${ }_{\text {on_pre- } \Omega}$ )

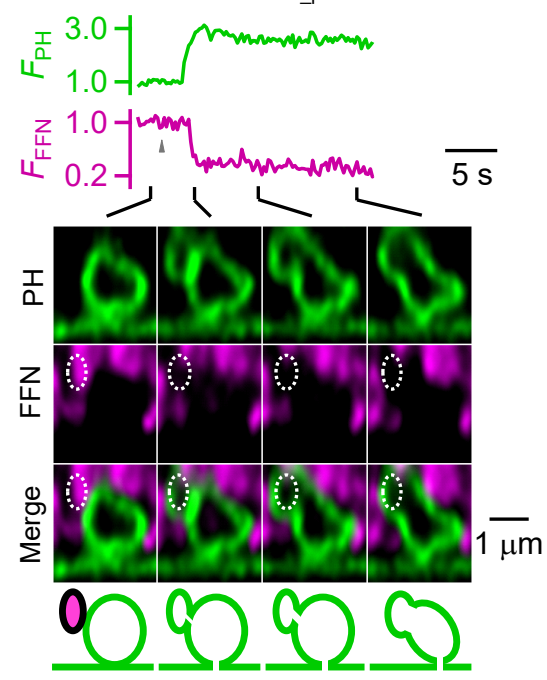

e
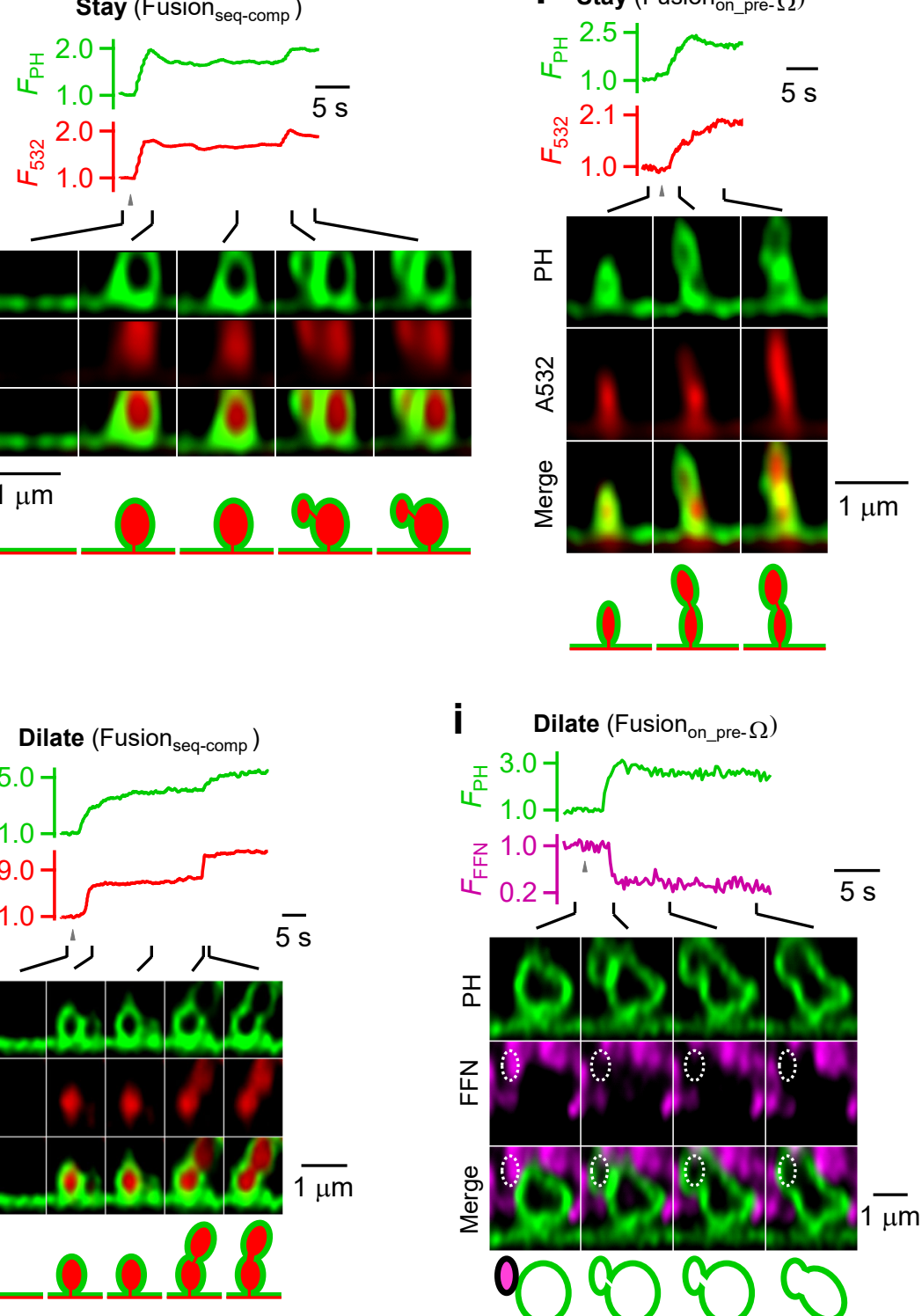
a Fusion $_{\text {seq-comp }}$

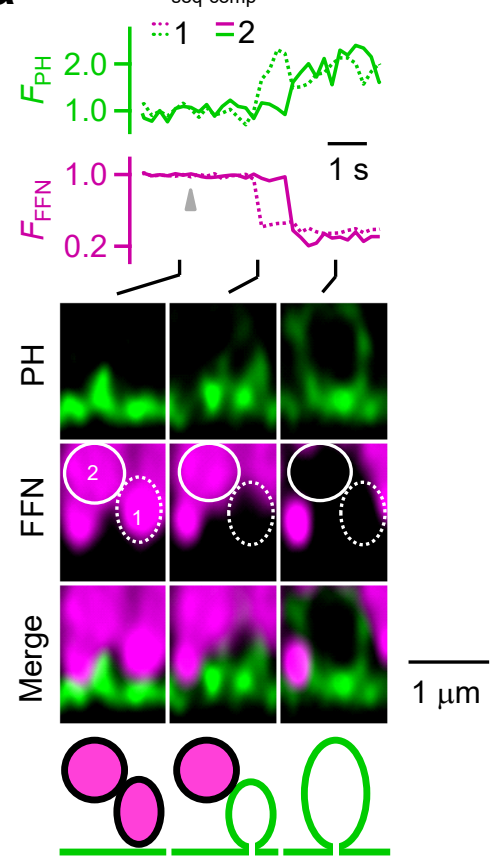

b

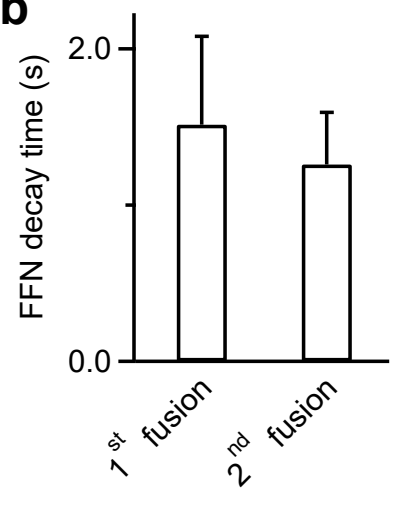

C
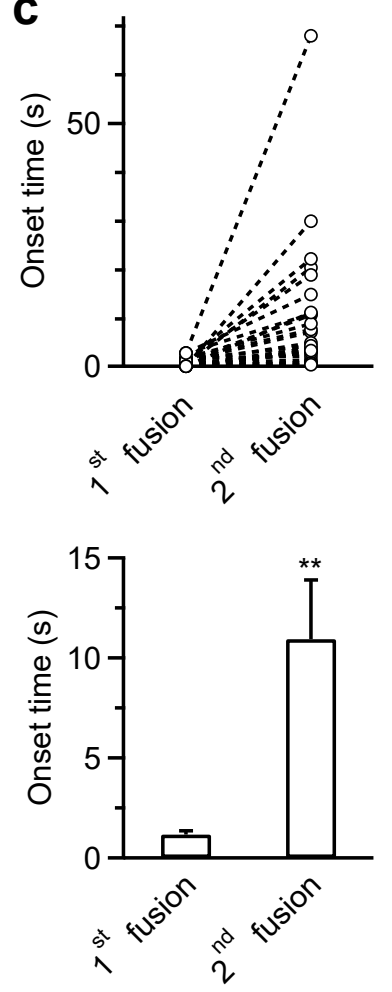

Figure 3 